\section{Hypoxaemia in COPD: looking beyond the lungs}

\section{Case history}

A 61-year-old woman was referred for further assessment of her chronic obstructive pulmonary disease (COPD). She had been diagnosed 5 years previously following a chest infection. Her symptoms consisted of an occasional cough and she had a UK Medical Research Council dyspnoea score of 3 out of 5. She denied haemoptysis or weight change. She had no significant past medical history with no background of atopy or childhood chest disease. Her father and mother both had winter bronchitis. She was an ex-smoker of 3 years and had a 37 pack-year history.

The patient had suffered one exacerbation in the previous year, had completed a pulmonary rehabilitation programme and was continuing with weekly maintenance classes.

She was being treated with Seretide (fluticasone/salmeterol) 500 Accuhaler (one inhalation b.i.d), Tiotropium $18 \mu \mathrm{g}$ once a day, Ventolin (salbutamol) as required and multivitamin tablets.

Her chest was hyperinflated with a few crepitations at the right lung base. Her body mass index was $23 \mathrm{~kg}$ per $\mathrm{m}^{2}$.
She had a forced expiratory volume in $1 \mathrm{~s}$ of $0.58 \mathrm{~L}(23.2 \%$ predicted) with a vital capacity of $2.66 \mathrm{~L}$. Her transfer factor for carbon monoxide corrected for haemoglobin was 19\% pred. Breathing air, her partial pressure for carbon dioxide $\left(\mathrm{PCO}_{2}\right)$ was $5.5 \mathrm{kPa}$ and arterial oxygen partial pressure was $7.16 \mathrm{kPa}$. Computed tomography showed homogenous emphysema.

A transthoracic echocardiogram was carried out.

\section{Task 1}

What echocardiographic measurements are important to note in COPD?
a) Right ventricular systolic pressure (RVSP)
b) Right ventricular (RV) dimensions
c) Tricuspid annular plane systolic excursion (TAPSE)
d) Pulmonary artery acceleration time

Z. Shaikh1

M.B. de Villa 2

N. Hopkinson ${ }^{3}$

1 Dept of Sleep and Ventilation, 2Echocardiography Dept and

3 National Heart and Lung Institute, Royal Brompton Hospital, London, UK

\section{Correspondence \\ Z. Shaikh}

Dept of Sleep and Ventilation

Royal Brompton Hospital

Fulham Road

London

SW3 6NP

UK

Fax: 442073518911

E-mail:z.shaikh07@

imperial.ac.uk

\section{Provenance}

Commissioned article, peer reviewed

Competing interests None declared 


\section{Answer 1}

a) This is a correct answer. RVSP in the absence of pulmonary stenosis is equal to systolic pulmonary artery pressure. The technique used to calculate this relies on detecting tricuspid regurgitation (TR) (figure 1). A degree of TR is present in most normal hearts. Doppler ultrasound is used to detect the velocity of this regurgitant jet (figure 2). This velocity reflects the pressure gradient or pressure drop between the RV and the right atrium. This pressure gradient can be calculated by putting the maximum velocity ( $V$ max) of blood flow measured by the Doppler trace into the Bernoulli equation:

$$
\text { Pressure gradient }=4 \times\left(V_{\max }\right)^{2}
$$

Thus, for a velocity of $2 \mathrm{~m}$ per $\mathrm{s}$

$$
\begin{aligned}
\text { Pressure gradient } & =4 \times 2^{2} \\
& =16 \mathrm{mmHg}
\end{aligned}
$$

The echo probe is positioned in multiple views so that TR can be imaged at various angles. The jet that gives the fastest velocity and therefore the highest pressure gradient is used to calculate RVSP. A velocity of 2-2.5 $\mathrm{m}$ per $\mathrm{s}$ is within normal limits. Right atrial pressure can be estimated by measuring the height of the jugular venous pressure (JVP). RVSP is then determined by:

$$
\mathrm{RVSP}=\text { pressure gradient }+ \text { right atrial pressure }
$$

Although this technique is simple and noninvasive, TR is often difficult to detect or the velocity is underestimated as COPD patients have limited acoustic windows because lung tissue obscures cardiac structures. Rightheart catheterisation therefore remains the current gold standard for the assessment of pulmonary artery pressure in COPD.

b) This is a correct answer. A dilated, poorly contracting right heart may indicate cor pulmonale. The RV wraps around the left ventricle forming an elliptical shape and single measurements cannot reflect its complex geometry. Precise endocardial definition is also difficult. All estimates of RV dimensions must therefore be interpreted with caution. A simple rule of thumb is if the RV is the same size or bigger than the left heart in the four-chamber view, then it is said to be dilated. Cardiac magnetic resonance imaging (MRI) provides greater accuracy in measurement of right ventricular size; however, this is not easily accessible and the cost of the test is often prohibitive.

c) This is a correct answer. Contraction of the RV occurs predominantly in the longitudinal plane with movement towards the cardiac apex in systole. Measurement of the distance that the tricuspid annulus moves during systole provides a simple, reproducible measure of RV contractile function (figure 3).

d) This is a correct answer. The pulmonary circulation is a low resistance circulation and hence blood flow through the RV outflow tract in systole resembles a symmetrical dome shape with equal acceleration and deceleration periods (figure 4). In the presence of increased pulmonary resistance, there is an early peak with a slower deceleration. The acceleration time is therefore shortened, closely resembling that of the systemic circulation.

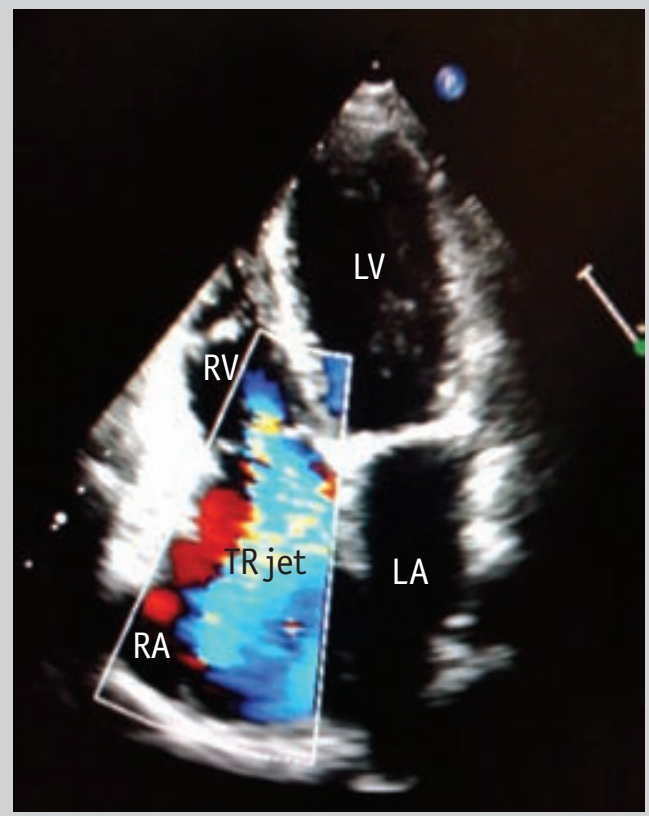

Figure 1

An example of a four-chamberview. The bright blue colour signal is a regurgitant jet of TR. This is severe $T R$, as it fills the right atrium (RA). LA: left atrium, LV: left ventricle.

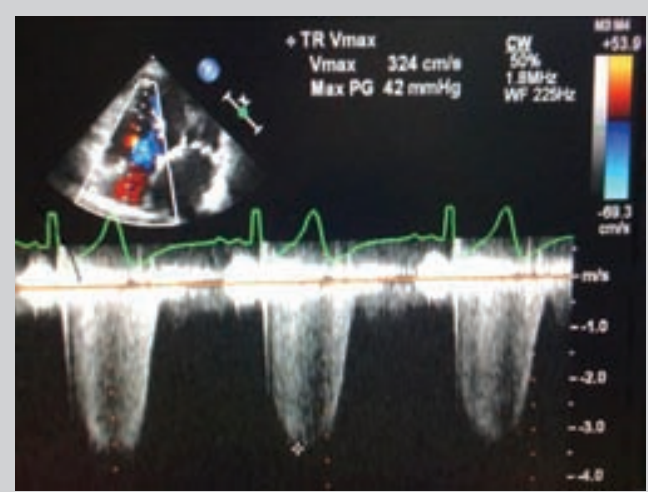

Figure 2

An example of a continuous-wave (CW) Doppler tracing of the velocity of blood flow in the jet of TR. The maximum velocity represents the maximum systolic pressure gradient between the right atrium and ventricle. In this example, it is $324 \mathrm{~cm}$ per s or $3.24 \mathrm{~m}$ per s. If this is put into the Bernouilli equation: pressure gradient $(P G)=$ $4 \times\left(V_{m a x}\right)^{2}$ or $4 \times(3.24)^{2}$, which is equal to $42 \mathrm{mmHg}$. RVSP or systolic pulmonary artery pressure is equal to 42 + height of JVP. 


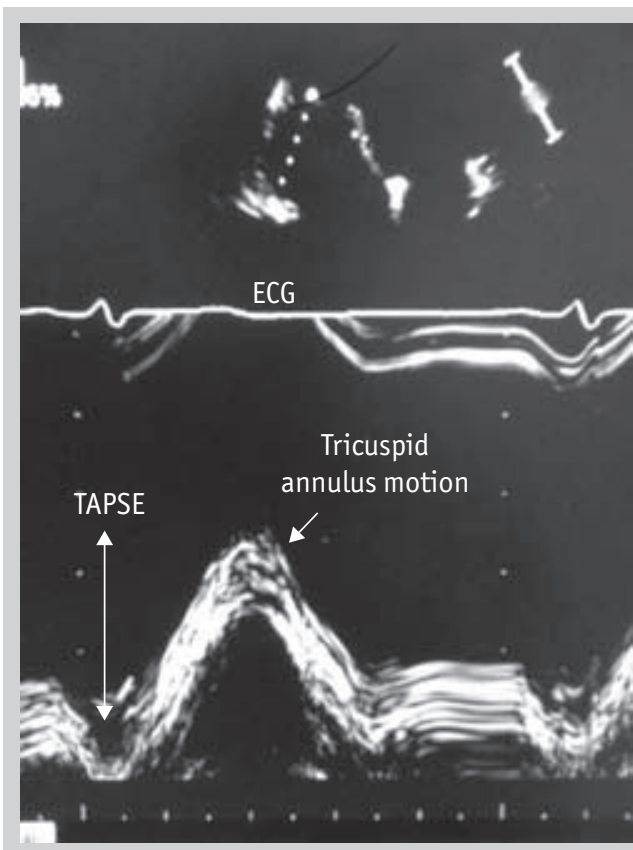

Figure 3

An M-mode recording showing tricuspid annulus motion throughout the cardiac cycle. The tricuspid annulus is a fibrous structure providing support for the tricuspid valve and dividing the right atrium and ventricle. The annulus moves towards the probe in systole, shown by the upward movement on the tracing. The height of the upward motion should be $>2 \mathrm{~cm}$. This measurement represents TAPSE.
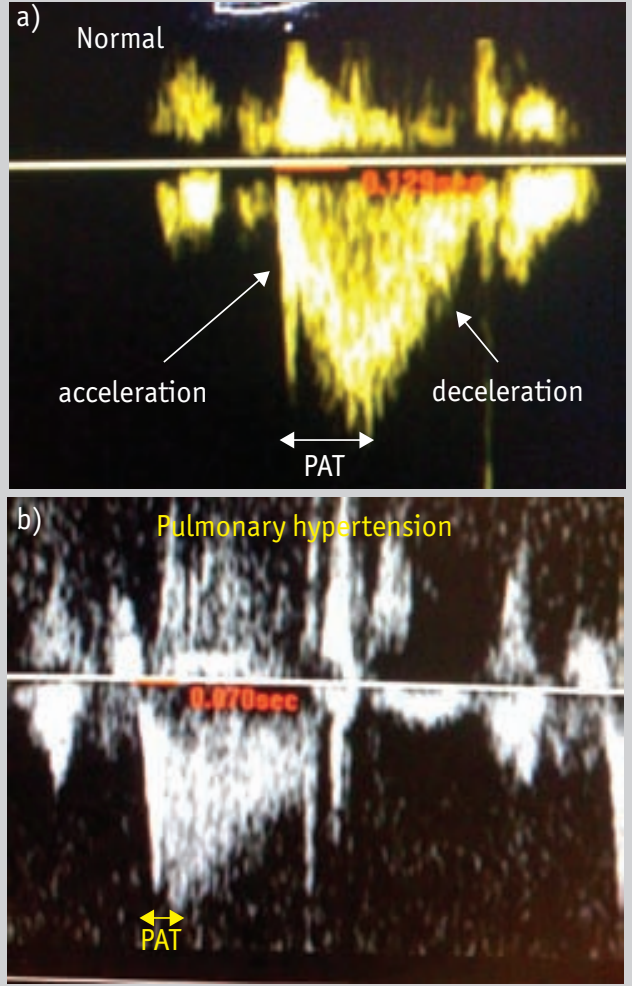

Figure 4

Doppler recordings of blood flow travelling through the pulmonary artery during systole. a) normal trace. There is a dome shape to the tracing with roughly equal acceleration and deceleration times. The time taken for blood flow to accelerate through the pulmonary valve (pulmonary acceleration time (PAT)) is normally $>120 \mathrm{~ms}$. In this case it is $129 \mathrm{~ms}$. b) taken from a patient with pulmonary hypertension and the PAT is $70 \mathrm{~ms}$.
The patient's echocardiogram demonstrated that she had normal left ventricular size and function. RV size and systolic function were also normal. There was insufficient TR to estimate RVSP. However, the pulmonary acceleration time was short suggesting increased pulmonary vascular resistance.

\section{Task 2 \\ The patient's oxygen saturations ranged between $91 \%$ at rest and $84 \%$ after dressing. Which cardiac causes could contribute to this drop in satu- ration with minimal exercise?
a) Patent ductus arteriosus (PDA)
b) Patent foramen ovale (PFO)
c) Left ventricular dysfunction
d) Secundum atrial septal defect (ASD) 


\section{Answer 2}

a) This is incorrect. A PDA is a persistent connection between the pulmonary artery and the aorta that forms a systemic-to-pulmonary shunt. Moderate-to-large PDAs significantly increase blood volume flowing through the pulmonary circulation. Untreated, this eventually leads to an increase in pulmonary vascular resistance, which, when elevated to systemic levels, reverses the direction of the shunt and leads to hypoxaemia. This defect usually presents in childhood and although it is eventually a cause of hypoxaemia, it is unlikely to be an incidental finding in this patient.

b) This is correct. In utero, the foramen ovale forms a flap-like connection between the two atria of the developing heart. It's function is to permit one-way flow from the right to left atrium. This allows oxygenated blood returning to the right atrium from the mother's placenta to bypass the dormant fetal lungs and enter the left atrium. At birth, lung inflation, decreasing pulmonary vascular resistance and initialisation of a pulmonary circulation result in a decrease in right atrial and an increase in left atrial pressure. This reverses the pressure gradient and forces the two halves of the atrial septum together, therefore sealing the foramen ovale shut. Incomplete fusion within the first 2 years of life creates a PFO, an interatrial connection which is the most common cause of a systemic right to left shunt [1]. Note that due to the flap like nature of PFO in the healthy adult, blood does not usually flow from left to right. This contrasts with a secundum ASD which is a physical hole in the atrium and not a flap. Blood therefore goes from high to low pressure forming a left to right shunt. The overall prevalence of PFO in an autopsy study of 965 normal adult hearts was $27.3 \%$ [2]. Recent studies have reported a higher prevalence of PFO in respiratory conditions including COPD $[3,4]$, obstructive sleep apnoea [5] and high-altitude pulmonary oedema [6]. The reported data suggest that PFO may contribute to hypoxaemia.

c) This is correct. Poor cardiac output aggravates ventilation/perfusion mismatch and reduces mixed venous saturations. Although data on the prevalence of left ventricular dysfunction in COPD is lacking, these conditions frequently occur together. We know from the patient's echocardiogram that her left ventricular function is normal.

d) This is correct. Blood flow through a secundum ASD is initially from left to right. This causes right heart dilatation due to volume overload. The echo would therefore demonstrate a dilated RV, and colour flow through the interatrial septum usually reveals left to right blood flow. Certain manoeuvres such as coughing and straining can temporarily reverse the direction of flow and potentially cause hypoxaemia.

\section{Task 3 \\ Which of the following additional tests would you perform? \\ a) Cardiac MRI \\ b) Echocardiogram with agitated saline contrast \\ c) Transcranial Doppler (TCD) with agitated saline contrast \\ d) $100 \%$ oxygen shunt study}




\section{Answer 3}

a) This is incorrect. In this particular clinical case, cardiac MRI is unlikely to provide any further useful clinical information that was not given by the echocardiogram.

b) This is correct. This is a useful investigation that can detect a PFO and also the presence of a pulmonary arteriovenous malformation. A solution containing saline, blood and air is initially agitated between two syringes to create a fine suspension of microbubbles.

These are reflected by ultrasound and hence make an excellent contrast agent. With the echo probe held in the four-chamber view, this mixture is injected into an antecubital vein at rest and following a valsalva manoeuvre. The solution can be seen to enter the right heart and it will cross to the left heart within 3-5 cardiac cycles if a PFO is present. The number of microbubbles seen in the left heart gives an indication of the size of the PFO (figure 5). If microbubbles appear in the left heart $>5$ cardiac cycles after entering the right heart, this is suggestive of a shunt at the pulmonary level.
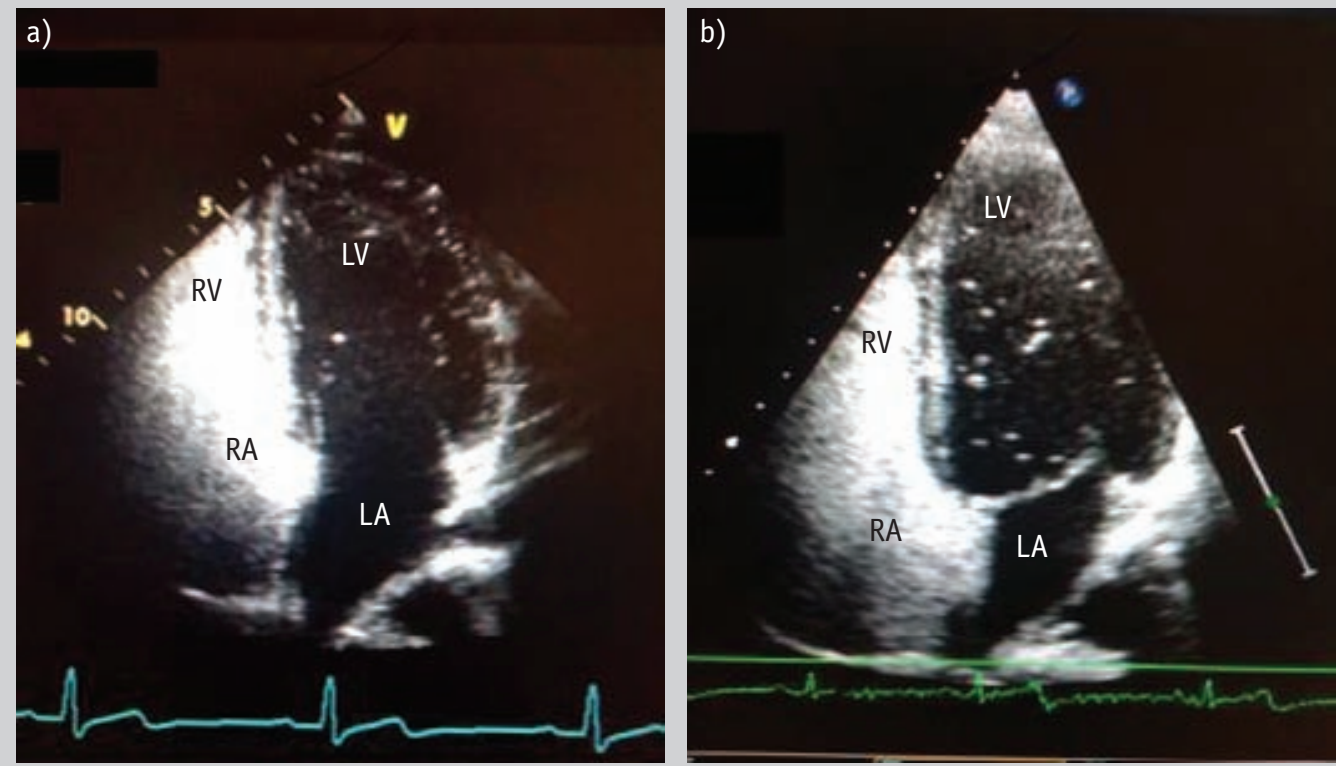

Figure 5

These four-chamber images are all taken from contrast studies. a) Bright, white contrast is seen in the right atrium $(R A)$ and $R V$; however no contrast is detected in the left heart. This patient does not have a PFO. b) A few microbubbles can be seen in the left ventricle (LV). This is a small PFO. c) Contrast is clearly detected in the left heart, but not enough to completely opacify any chamber. This is a moderate-size PFO. d) Contrast can be seen in all four chambers, completely filling the left heart. This is a large PFO. $L A$ : left atrium.
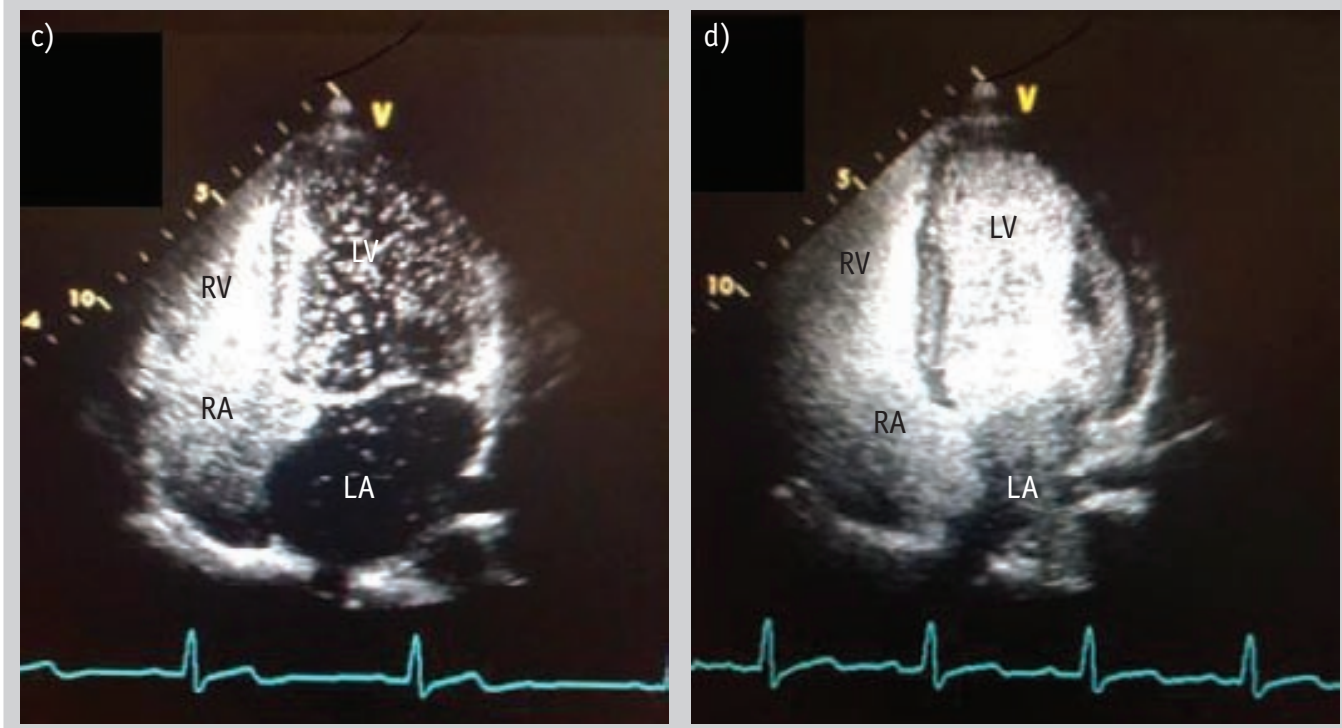

c) This is correct. Contrast TCD will detect a right to left shunt in poorly echogenic patients such as those with COPD. The system consists of two ultrasound probes that are used to insonate the middle cerebral artery bilaterally through the temporal bone windows. The probes are secured using an adjustable head frame. The Doppler signal gain depth and gate size is adjusted to detect flow in both middle cerebral arteries (figure 6). Agitated saline contrast is injected. If there is no PFO, the microbubbles will enter the right heart and travel to the pulmonary circulation where they will either dissolve or be filtered out. If there is a PFO, the bubbles will cross the interatrial septum and enter the systemic circulation, and these are detected in the cerebral arteries by the TCD machine. This simple technique also permits semi-quantification of embolic contrast material [7].

d) This is correct. The shunt fraction can be assessed using the 100\% oxygen method. The patient is asked to breathe $100 \%$ oxygen for 15-20 min. Arterial blood gases are taken and the percentage of blood that is anatomically shunted can be calculated. A shunt fraction $>5 \%$ is abnormal. Although this helps to detect a shunt, it provides no information on its location. 


\section{Figure 6}

These images are of a contrast transcranial Doppler recording, at rest (a) and following a valsalva manoeuvre (b). The bright signals in b) represent bubble contrast arriving in the middle cerebral artery. LMCA: left middle cerebral artery; RMCA: right middle cerebral artery; depth: depth of Doppler sample volume in $\mathrm{mm}$; power: transmitting Doppler output power; mean: mean cerebral blood flow velocity in $\mathrm{cm}$ pers. a)
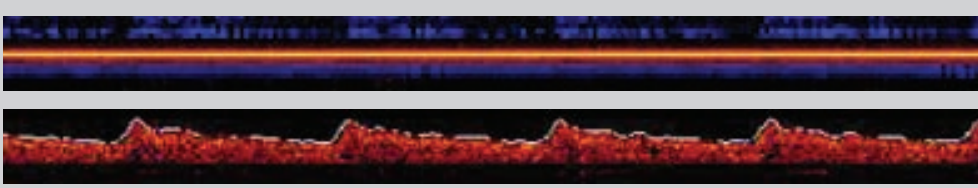

RMCA

Depth: $56 \mathrm{~mm}$

Power: $30 \%$

Mean: $60 \mathrm{~cm} / \mathrm{s}$

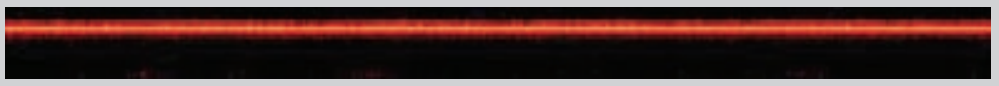

LMCA

Depth: $42 \mathrm{~mm}$

Whased

Power: $10 \%$

Mean: $45 \mathrm{~cm} / \mathrm{s}$

b)

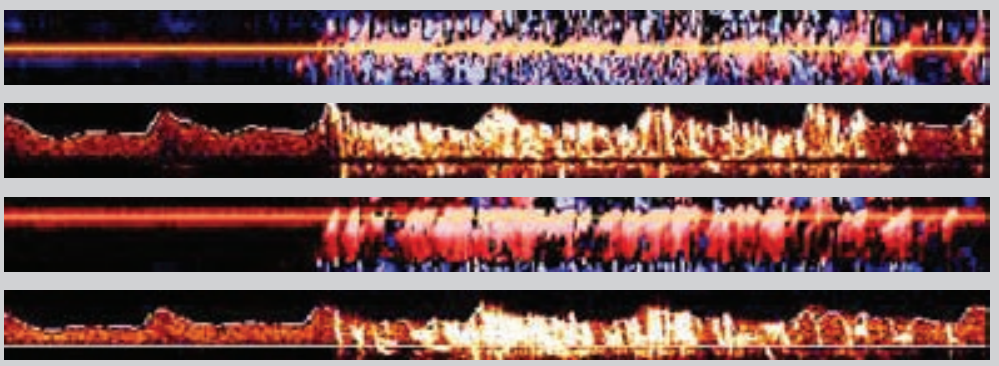

RMCA

Depth: $56 \mathrm{~mm}$

Power: $30 \%$

Mean: $57 \mathrm{~cm} / \mathrm{s}$

LMCA

Depth: $42 \mathrm{~mm}$

Power: $10 \%$

Mean: $44 \mathrm{~cm} / \mathrm{s}$
The patient underwent a contrast echocardiogram. At rest, she was found to have a small-size PFO which became moderate in size following a valsalva manoeuvre. She also underwent a TCD study, which confirmed the echocardiographic findings (figure 7).

In order to determine the amount of right to left shunting through the PFO during exercise, the patient underwent a contrast TCD study during exercise (table 1). Interestingly, the number of microemboli detected by TCD tripled during exercise.
Task 4

What treatment would you advise?

a) Long-term oxygen therapy

b) PFO closure

c) Diuretics

d) Vasodilators

Table 1 Degree of right to left shunting as measured by contrast transcranial Doppler during exercise on a cycle ergometer

\begin{tabular}{|c|c|c|c|c|c|c|c|}
\hline $\begin{array}{l}\text { Time } \\
\text { min }\end{array}$ & $\begin{array}{c}\text { Load } \\
\text { W }\end{array}$ & $\begin{array}{c}\text { Number of } \\
\text { microemboli } \\
\text { detected by TCD }\end{array}$ & $\begin{array}{l}\text { Size of } \\
\text { right to left } \\
\text { shunt }\end{array}$ & $\begin{array}{c}\text { Oxygen } \\
\text { saturation \% }\end{array}$ & $\begin{array}{c}V^{\prime} 0_{2} L \\
\text { per min }\end{array}$ & $\begin{array}{l}V^{\prime} \mathrm{CO}_{2} \mathrm{~L} \\
\text { per min }\end{array}$ & $\begin{array}{c}V^{\prime} E L \\
\text { per min }\end{array}$ \\
\hline Baseline & 0 & 17 & Small & 96 & 0.23 & 0.21 & 11.7 \\
\hline 2 & 5 & 45 & Moderate & 96 & 0.31 & 0.27 & 11.8 \\
\hline 4 & 25 & 34 & Moderate & 91 & 0.43 & 0.33 & 13.2 \\
\hline 6 & 45 & 75 & Moderate & 82 & 0.7 & 0.63 & 23.2 \\
\hline 7 & 0 & 50 & Moderate & 88 & 0.57 & 0.54 & 20.0 \\
\hline 10 & 0 & 42 & Moderate & 96 & 0.38 & 0.41 & 16.8 \\
\hline 14 & 0 & 30 & Small & 95 & 0.27 & 0.27 & 12.6 \\
\hline 20 & 0 & 30 & Small & 96 & 0.22 & 0.17 & 8.3 \\
\hline
\end{tabular}


During normal respiration

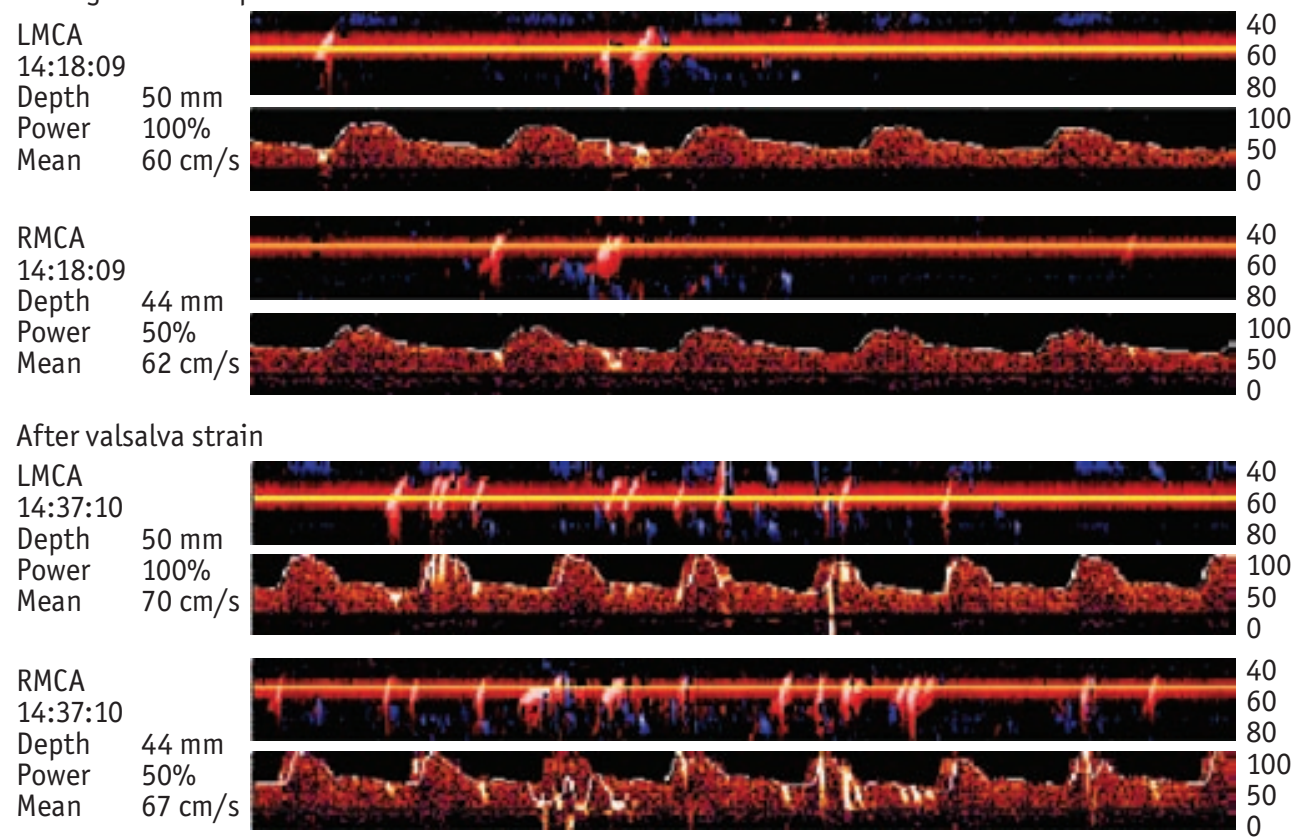

\section{Answer 4}

a) This is correct. Currently, long-term oxygen therapy is the only treatment offering mortality benefit in severe, hypoxaemic COPD $[8,9]$. By lowering pulmonary artery pressure, it may also reduce right to left shunting through a PFO. The patient was started on ambulatory oxygen therapy and has noticed improvements in her functional status.

b) This is incorrect. There is currently no evidence to suggest that PFO closure is of benefit in patients with COPD. Despite the findings of increased shunting on exercise, it was felt that the PFO did not contribute enough to warrant the invasive interventional procedure to close the PFO.

c) This is incorrect. Clinically, the patient was euvolaemic and there was no echocardiographic evidence of right ventricular dysfunction. Diuretics are therefore not advised.

e) This is incorrect. There is insufficient evidence from the echocardiogram performed to suggest persistently elevated pulmonary artery pressures. There is also no evidence that vasodilators are of benefit in COPD.

\section{Discussion}

This case highlights an extrapulmonary cause of hypoxaemia in COPD. To date, two observational studies have been published which, taken together, suggest that there is a higher prevalence of PFO in severe COPD $[3,4]$. The first study examined 20 patients with severe COPD and 20 agematched controls. A PFO was identified in $70 \%$ of the COPD group versus 35\% in controls [3] The second investigated 52 COPD patients and 50 age-matched controls [4]. A PFO was detected in $44 \%$ of the COPD group and $20 \%$ of the control group. 11 COPD patients (21\%) had a PFO with shunting at rest compared with none in the control group. The authors reported lower resting oxygen saturations in patients with COPD and a PFO than in those without (87.7\% versus $91.7 \%$; $\mathrm{p}<0.01$ ) and also suggested a "dose response" with the lowest saturations in the group with a PFO and shunting at rest $(84.1 \% ; p<0.001)$. The implications of the data are limited as patients were poorly characterised and investigators were not blinded; however it raises the question: does right to left shunting, particularly at rest through large PFO, contribute to hypoxaemia?

Given the patient's desaturation with minimal exercise and the absence of significant shunting through the PFO at rest, an exercise test was performed to determine the functional impact of the PFO. In the normal healthy population, right to left shunting during exercise is unlikely as left atrial pressure exceeds right atrial pressure. In COPD, there are several factors that lead to a reversal of this pressure gradient. First, during exercise, pulmonary vascular resistance and pulmonary artery pressure rise $[10,11]$. RV end-diastolic volume increases; however, RV ejection fraction remains static $[12,13]$. The exercise-induced increase in RV preload and afterload lead to an elevated right atrial pressure. In the presence of a PFO,
Figure 7

Contrast TCD recording from patient at rest (a) and after valsalva manoeuvre (b). The bright signals in b) represent bubble contrast arriving in the middle cerebral artery. The number of microemboli reaching the middle cerebral arteries following a valsalva manoeuvre doubled (moderate-size shunt) compared with the number detected at rest (small shunt). LMCA: left middle cerebral artery, RMCA: right middle cerebral artery; depth: depth of Doppler sample volume in mm; power: transmitting Doppler output power; mean: mean cerebral blood flow velocity in $\mathrm{cm}$ pers. 
this results in right to left shunting. It is thought that this offloads the right heart by acting as a pressure-release valve and maintains cardiac output albeit at the expense of systemic desaturation.

Secondly, abdominal muscle recruitment during exercise is common in patients with COPD [14]. Contraction of these muscles in expiration may lead to pleural pressures $>40 \mathrm{cmH}_{2} \mathrm{O}$, effectively producing a valsalva manoeuvre. The more negative inspiratory pressures would increase venous return and could promote right to left shunting.

Thirdly, because lung emptying in COPD requires more time due to the effects of increased airway resistance and reduced elastic recoil; gas trapping and dynamic hyperinflation is a well recognised phenomenon. Because the chest wall-cannot fully recoil towards functional residual capacity, there is continuous intrinsic positive expiratory pressure. Again, this mimics a valsalva manoeuvre. Higher lung volumes will produce greater elastic load and thus more negative inspiratory pressure. This will promote venous return, deliver a volume load to the RV and potentially increase right to left shunting through a PFO. Right to left shunting results in the addition of deoxygenated, acidaemic, and $\mathrm{CO}_{2}$-rich blood to the systemic circulation. In order to maintain $\mathrm{pH}$ and arterial $\mathrm{PCO}_{2}$ homeostasis, arterial chemoreceptors in the carotid bodies are stimulated to increase ventilation. This would in turn increase dynamic hyperinflation and cause further shunting.

In the absence of evidence of benefit, the patient did not undergo PFO closure. Several case reports have described percutaneous PFO closure specifically to control hypoxaemia and demonstrate immediate improvement in arterial oxygen tensions and dyspnoea following closure [15-20]. However, evidence from larger trials is lacking, and the physiological impact of PFO closure is uncertain. If a PFO acts as pressure-release valve, could closure have a negative effect, particularly on right ventricular function? This is an exciting area where further research is needed. A contrast echocardiogram is a simple, useful investigation that not only provides important information about cardiac function and pulmonary pressures, but may help shed light on why some COPD patients are more hypoxaemic than others who have a similar degree of lung function impairment.

\section{References}

1. Hara H, Virmani R, Ladich E. Patent foramen ovale: current pathology, pathophysiology, and clinical status. J Am Coll Cardiol 2005; 46: 1768-1776.

2. Hagen PT, Scholz DG, Edwards WD. Incidence and size of patent foramen ovale during the first 10 decades of life: an autopsy study of 965 normal hearts. Mayo Clin Proc 1984; 59: 17-20.

3. Soliman A, Shanoudy H, Liu J, Russell DC, Jarmukli NF. Increased prevalence of patent foramen ovale in patients with severe chronic obstructive pulmonary disease. J Am Soc Echocardiogr 1999; 12: 99-105.

4. Hacievliyagil SS, Gunen H, Kosar FM, Sahin I, Kilic T. Prevalence and clinical significance of a patent foramen ovale in patients with chronic obstructive pulmonary disease. Respir Med 2006; 100: 903-910.

5. Shanoudy H, Soliman A, Raggi P, Liu JW, Russell DC, Jarmukli NF. Prevalence of patent foramen ovale and its contribution to hypoxemia in patients with obstructive sleep apnea. Chest 1998; 113: 91-96.

6. Allemann Y, Hutter D, Lipp E, et al. Patent foramen ovale and high-altitude pulmonary edema. JAMA 2006; 296: 2954-2958.

7. Job FP, Ringelstein EB, Grafen Y, et al. Comparison of transcranial contrast Doppler sonography and transesophageal contrast echocardiography for the detection of patent foramen ovale in young stroke patients. Am J Cardiol 1994; 74: 381-384.

8. Long term domiciliary oxygen therapy in chronic hypoxic cor pulmonale complicating chronic bronchitis and emphysema. Report of the Medical Research Council Working Party. Lancet 1981; 1: 681-686.

9. Nocturnal Oxygen Therapy Trial Group. Continuous or nocturnal oxygen therapy in hypoxemic chronic obstructive lung disease: a clinical trial. Ann Intern Med 1980; 93: 391-398.

10. Schonhofer B, Barchfeld T, Wenzel M, Köhler D. Long-term effects of non-invasive mechanical ventilation on pulmonary haemodynamics in patients with chronic respiratory failure. Thorax 2001; 56: 524-528.

11. Raeside DA, Brown A, Patel KR, Welsh D, Peacock AJ. Ambulatory pulmonary artery pressure monitoring during sleep and exercise in normal individuals and patients with COPD. Thorax 2002; 57: 1050-1053.

12. Mahler DA, Brent BN, Loke J, Zaret BL, Matthay RA. Right ventricular performance and central circulatory hemodynamics during upright exercise in patients with chronic obstructive pulmonary disease. Am Rev Respir Dis 1984; 130: 722-729.

13. Light RW, Mintz HM, Linden GS, Brown SE. Hemodynamics of patients with severe chronic obstructive pulmonary disease during progressive upright exercise. Am Rev Respir Dis 1984; 130: 391-395.

14. Kyroussis D, Polkey MI, Hamnegård CH, Mills GH, Green M, Moxham J. Respiratory muscle activity in patients with COPD walking to exhaustion with and without pressure support. Eur Respir J 2000; 15: 649-655.

15. Mottram PM, McGaw DJ, Meredith IT, Peverill RE, Harper RW. Profound hypoxaemia corrected by PF0 closure device in carcinoid heart disease. Eur J Echocardiogr 2008; 9: 47-49.

16. Kuch B, Riehle M, von Scheidt W. Hypoxemia from right-to-left shunting through a patent foramen ovale in right ventricular infarction: treatment by revascularization, preload reduction, and, finally, interventional PF0 closure. Clin Res Cardiol 2006; 95: 680-684.

17. Shnaider $\mathrm{H}$, Shiran A, Lorber A. Right ventricular diastolic dysfunction and patent foramen ovale causing profound cyanosis. Heart 2004; 90: e31.

18. Allan JJ, Marinelli C, Dellsperger KC, Winniford MD. Percutaneous balloon catheter closure of a patent foramen ovale in a patient with pulmonary disease, profound hypoxemia, and normal right heart pressures. Clin Cardiol 1997; 20: 307-309.

19. Remy-Jardin, M, Remy J, Wallaert B. Right-to-left shunting through a patent foramen ovale without pulmonary hypertension. Transient improvement after balloon catheter closure. Chest 1990; 97: 1250-1252.

20. Piechaud JF. Hypoxemia related to right-to-left shunting through a patent foramen ovale: successful percutaneous treatment with the CardioSeal device. J Interv Cardiol 2001; 14: 57-60. 